\title{
Comparison of different numerical approaches to the 1D sea-ice thermodynamics problem
}

\author{
Frederic Dupont ${ }^{\mathrm{a}, *}$, Martin Vancoppenolle ${ }^{\mathrm{b}}$, Louis-Bruno Tremblay ${ }^{\mathrm{c}}$, Hendrik Huwald ${ }^{\mathrm{d}}$ \\ ${ }^{a}$ MSC, Environment Canada, Dorval, QC, Canada

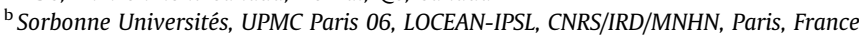 \\ ${ }^{\mathrm{C}}$ Atmospheric and Oceanic Sciences Dept, McGill U., Montreal, QC, Canada \\ ${ }^{\mathrm{d}}$ School of Architecture, Civil and Environmental Engineering, Ecole Polytechnique Fédérale de Lausanne (EPFL), Lausanne, Switzerland
}

\section{A R T I C L E I N F O}

\section{Article history:}

Received 13 June 2014

Received in revised form 11 December 2014

Accepted 15 December 2014

Available online 8 January 2015

\section{Keywords:}

1D sea-ice thermodynamics

Sigma vertical coordinate

Enthalpy conserving numerical schemes

Mode comparison

\begin{abstract}
A B S T R A C T
The vertical one-dimensional sea-ice thermodynamic problem using the principle of conservation of enthalpy is revisited here using (1) the Bitz and Lipscomb (1999) finite-difference approach (FD), (2) a reformulation of the sigma-level transformation of Huwald et al. (2005b) (FV) and (3) a Finite Element approach also in sigma coordinates (FE). These three formulations are compared in terms of physics, numerics, and performance, in order to identify the best choice for large-scale climate models. The BL99 formulation sequentially treats the diffusion of heat and the changes in the vertical position of the ice-snow layers. In contrast, the FV sigma-level transformation elegantly treats both simultaneously. The original FV formulation suffers however from slow convergence. The convergence can nonetheless be improved significantly with a few simple modifications to the original code. The three formulations are compared following the experimental protocol of the Sea Ice Model Intercomparison Project for ice thermodynamics (SIMIP2). It is found that all formulations converge to the same solution. The FD approach, however, suffers from the added cost of the remapping step at large number of ice layers we include in the appendix an optimized version of the FD code-written by one of the reviewer-that resolves this issue. Finally the FE formulation results in a sub-surface temperature over-estimation at low resolution, a problem which disappears at high resolution. Hence, only FD and FV are found suitable for climate models.
\end{abstract}

Crown Copyright $\odot 2014$ Published by Elsevier Ltd. All rights reserved.

\section{Introduction}

The representation of thermodynamics, central to large-scale sea ice models (Washington and Parkinson, 2005), advances in time the vertical temperature profile based on a non-linear heat equation. The sea ice thermal properties depend on salinity and temperature, mirroring changes in the volume of interstitial brine (see Hunke et al. (2011), for a review). Including these non-linear thermal properties in large-scale sea ice models was long hindered by the non-conservation of heat in the standard Maykut and Untersteiner (1971) formulation of sea ice thermodynamics, a problem that was fixed by Bitz and Lipscomb (1999, hereafter BL99). The present paper focuses on the non-trivial numerical challenges associated with solving the non-linear heat equation in sea ice.

\footnotetext{
* Corresponding author.

E-mail addresses: frederic.dupont@ec.gc.ca (F. Dupont), martin.vancoppenolle@locean-ipsl.upmc.fr (M. Vancoppenolle), bruno.tremblay@mcgill.ca (L.-B. Trem-
} blay), hendrik.huwald@epfl.ch (H. Huwald).
The main non-linearity in the sea ice heat equation is associated with the latent heat absorption and release due to the ice freezing or dissolution at the edge of brine inclusions (Semtner, 1984; Ebert and Curry, 1993; Bitz and Lipscomb, 1999; Vancoppenolle et al., 2005). This corresponds to an extra term in the sea ice specific heat capacity (Malmgren, 1927; Untersteiner, 1961; Ono, 1967), which in turn, following brine volume fraction, depends on salinity and temperature:

$c=c_{0}-L_{0} \frac{T_{f}}{T^{2}}$.

$c_{0}$ is the pure ice specific heat capacity, $L_{0}$ is the pure ice latent heat of fusion, $T$ the ice temperature $\left({ }^{\circ} \mathrm{C}\right)$ and $T_{f}=-\mu \mathrm{S}$, the freezing point of sea water at a given salinity, with $S$ the macroscale (bulk) ice salinity in $\mathrm{g} / \mathrm{kg}$ (following IOC et al., 2010) and $\mu=0.054 \mathrm{~K}(\mathrm{~g} /$ $\mathrm{kg})^{-1}$. Following BL99, $T_{f}$ can also represent the liquidus temperature in the brine inclusions. Hence, given a certain ice temperature, the brine salinity can be retrieved using this relation. However, in order to avoid confusion, we limit $T_{f}$ to the freezing point of sea 
water hereafter. Near $T_{f}$, the extra term in Eq. (1) reaches values that are at least one order of magnitude larger than the pure ice specific heat, affecting the mean equilibrium thickness and the phase of its seasonal cycle (BL99; Vancoppenolle et al., 2005).

A numerical implementation of the non-linear heat equation in sea ice should conserve energy. This can be easily achieved if the sea ice thermodynamic formulation is based on an expression of enthalpy $(E)$ that is consistent with the specific heat (BL99):

$E\left(T, T_{f}\right)=c_{0}\left(T-T_{f}\right)-L_{0}\left(1-\frac{T_{f}}{T}\right)+c_{w} T_{f}$,

where $c_{w}$ is the specific heat capacity of fresh water. For numerical purposes, it is worth noting that the enthalpy conservation can be exactly ensured for discrete-in-time systems

$$
\begin{aligned}
E\left(T^{\prime}, T_{f}\right)-E\left(T, T_{f}\right) & =\int_{T}^{T^{\prime}} c d T=\left(c_{0}-L_{0} \frac{T_{f}}{T T^{\prime}}\right)\left(T^{\prime}-T\right) \\
& =c^{\prime}\left(T^{\prime}-T\right) .
\end{aligned}
$$

where $T$ and $T^{\prime}$ are initial and final temperatures, respectively. Because of the presence of non-linear terms (such as $c^{\prime}$ ), an iterative non-linear solver must be used in order to obtain an enthalpy-conserving numerical solution of the heat equation.

The first enthalpy-conserving implementation of the non-linear equation of heat is the BL99 formulation. Based on a $z$-coordinate system, it separates the sea ice thermodynamic iteration in three steps: (1) iteration of the heat equation (temperature profile), (2) computation of the accretion/ablation rates, modification of ice thickness, and (3) conservative remapping of the enthalpy (temperature) on the new grid accounting for the change in thickness. This BL99 approach, implemented in CICE (Hunke and Lipscomb, 2010) and LIM3 (Vancoppenolle et al., 2009) models will be somewhat arbitrarily referred to as Finite Difference (FD).

Huwald et al. (2005a, hereafter HTB05), reformulate the problem with a vertical sigma coordinate system, which introduces an internal "regridding" vertical velocity related to the changing ice and snow thicknesses. The latter approach solves in the same iterative loop both the non-linear thermodynamics and the vertical remapping due to the changes in thickness, what is otherwise treated in three steps in FD. Even though in practice the differences between the two approaches are small, that of HTB05 has a definite appeal since the heat equation can directly 'respond' to the regridding. Because the HTB05 method applies the sigma coordinate system, we will refer to it as a Finite Volume (FV) scheme, in analogy to sigma-coordinate ocean models such as the Princeton Ocean Model (Mellor and Blumberg, 1985).

In the FD or FV methods, since the enthalpy and temperature are intertwined for conservation reasons, the temperature is defined at the center of each model layer. The surface and bottom energy balance requires however a temperature value at the top and bottom surfaces. Variables for both interfacial temperatures are therefore added to the otherwise layer-centered discretization, complicating the discretization by the introduction of half distances and off-centered differencing. We propose a simple linear Finite Element (FE) approach to simplify this: the temperature is continuous between layers of different physical properties but the slope of the temperature is not (for instance at the ice-snow interface). Therefore, the temperature profile naturally fits the discontinuity in the properties of the medium and also fits the requirement of the energy balance at the extremities of the medium. We show that this third method expressed in sigma-coordinate can also yield an enthalpy-conserving scheme and similar results to the other two methods.

In the same spirit of the Sea-Ice Model Intercomparison Project (SIMIP) for the sea-ice dynamical part (Kreyscher et al., 2000), a protocol was proposed for comparing the sea-ice thermodynamics, also known as SIMIP2 (HTB05). This is based on the observations taken during the Surface Heat Budget of the Arctic (SHEBA) Ocean Project (Perovich et al., 1999; Persson et al., 2002). As in HTB05, this protocol is used to compare the three methods with respect to physical and numerical criteria.

Although salinity exhibits a clear non-uniform vertical profile through the ice during the SHEBA experiment (varying from $1 \mathrm{~g} / \mathrm{kg}$ at the surface to $4 \mathrm{~g} / \mathrm{kg}$ at the base; Perovich and Elder, 2002), the salinity is considered here constant and uniform at $4 \mathrm{~g} / \mathrm{kg}$. A vertically-varying profile would certainly improve the realism of the different simulations. However, the main focus of this contribution being the intercomparison of different numerical formulations of ice thermodynamics, we fear that introducing more complexities would compromise our ability to draw conclusions of the comparative performances of the different models. As such, our goal is not to reproduce in detail the observed fields, but rather to define a background state that can be compared to different thermodynamic sea-ice models. The simpler this background state, the easier and comprehensive our analysis will be.

The paper is divided in the following sections: the development of the equations for fixed level and generalized sigma coordinate (Section 2), their discretized forms for the FD (Section 3), the FV (Section 4) and FE (Section 5) models, the different models' evaluation based on the SIMIP2 protocol (Section 6), and the discussion and conclusions (Section 7).

\section{General equation for enthalpy conservation}

We now derive the set of equations in their continuous form used for the two coordinate systems, starting first with the fixedlevel $z$-coordinate system. Starting from the enthalpy conservation through ice and snow (see BL99), we write:

$\left.\rho c \frac{\partial T}{\partial t}\right|_{z}=\left.\rho \frac{\partial E}{\partial t}\right|_{z}=\frac{\partial}{\partial z}\left(k \frac{\partial T}{\partial z}+R\right)$,

where $\rho$ is the density of the ice or snow, $t$ the time, $R$ the amount of shortwave radiation penetrating into the medium, $R=I_{0} \exp \int_{z}^{z_{s u}} \kappa d z$ with $\kappa$ being the vertical extinction coefficient for short-wave radiation, $I_{0}$ the fraction of incident shortwave radiation below the surface scattering layer and $z$ is the vertical axis, positive upward and expressed in meters. $z_{s u}$ defines the coordinate value at the top surface ( $b$ and su subscripts refer respectively to the bottom and top surfaces). $k$ is the conductivity computed following Pringle et al. (2007) in the ice, constant in the snow at $0.31 \mathrm{~W} \mathrm{~m}^{-1} \cdot \mathrm{K}^{-1}$.

We now introduce the moving vertical coordinate. The simplest choice is the sigma coordinates where $\sigma=\frac{z-z_{b}}{z_{s u}-z_{b}}$, with $z_{s u}-z_{b}=h$ being the ice thickness (the same can be done in the snow, but for the time being, we will focus on the ice representation). $z$-space inside the ice is then mapped linearly onto $\sigma$-space where $\sigma$ varies between 0 and 1 . Hence, the left hand side of the Eq. (4) can be rewritten in terms of $\sigma$

$\rho\left(\left.\frac{\partial E}{\partial t}\right|_{\sigma}+\dot{\sigma} \frac{\partial E}{\partial \sigma}\right)=\frac{\partial}{\partial z}\left(k \frac{\partial T}{\partial z}+R\right)$,

where $\dot{\sigma}$ is the vertical grid advection velocity in the sigma-coordinate system. The HTB05 discretization takes advantage of the local continuity equation expressed in generalized sigma-coordinate:

$\frac{\partial(\partial z / \partial \sigma)}{\partial t}+\frac{\partial(\partial z / \partial \sigma \cdot \dot{\sigma})}{\partial \sigma}=0$.

Note that the terms involving $\dot{\sigma}$ can be written in terms of $\omega=\dot{\sigma}(\partial z / \partial \sigma)$ in Eqs. (5) and (6), a vertical velocity with units of $\mathrm{m} / \mathrm{s}$. $\omega$ is easily understood as a volume transport into (out of) a grid cell as ice grows (melts). If Eq. (6) is integrated over the whole 
column in $\sigma$ space, we obtain the rate of change of the total ice thickness $h$ :

$\frac{\partial h}{\partial t}=\int_{0}^{1} \frac{\partial(\partial z / \partial \sigma)}{\partial t} d \sigma=-\int_{0}^{1} \frac{\partial \omega}{\partial \sigma} d \sigma=-[\omega]_{0}^{1}=\omega_{b}-\omega_{s u}$.

If $\omega_{b}$ is positive at the base, ice accumulates at the base (freezing). If $\omega_{s u}$ is positive at the top, ice or snow is melting away at the top surface, hence the equivalence between $\omega$ and a transport into or out of the whole column or a single layer. It is also apparent that $\omega$ is simply linear in $z$ from the boundary values, to zero at the ice-snow interface when present.

Multiplying Eq. (5) by $\frac{\partial z}{\partial \sigma}$ and combining with Eq. (6) leads to the local conservation of enthalpy in presence of a varying local thickness (here $\rho$ is constant for each medium, i.e. ice or snow)

$\frac{\partial(\rho E \partial z / \partial \sigma)}{\partial t}+\frac{\partial(\rho E \omega)}{\partial \sigma}=\frac{\partial}{\partial \sigma}\left(k \frac{\partial T}{\partial z}+R\right)$.

The boundary condition consists of including the rate of ablation and accretion based on the internal heat conductive $k \frac{\partial T}{\partial z}$ and net downward $F$ fluxes at the upper and lower surfaces following Schmidt et al. (2004)

$\left[\rho E \frac{d z}{d t}=F-k \frac{\partial T}{\partial z}\right]_{z=z_{b}, z_{s u}}$,

where $F$ is positive oriented downward and $d z / d t$ is the rate of change in a level of a given top or bottom surface. In effect, $d z / d t$ can be replaced, following our convention, by $\omega$. At the top surface, $F_{s u}$ denotes the short and long wave radiation, turbulent sensible and latent heat flux, positive when warming the ice. The heat flux at the bottom $F_{b}$ represents the ocean-ice turbulent sensible heat exchange. Following the same convention, $F_{b}$ is positive when warming the ocean. The temperature at the base is considered fixed at the freezing point of the surface ocean whereas the top surface temperature is derived from the top boundary condition.

\section{Equation for the fixed-depth coordinate model using finite differences (FD)}

The continuous form of the equations are now discretized, starting with those related to the fixed-level $z$-coordinate system. We start from the original heat equation in $z$-coordinate (Eq. (4)) which is discretized using Finite Differences (FD), following the BL99 method. The ice thickness is divided into $N$ layers of equal thickness. The temperature $T_{i}$ is taken at mid-layer depth and $\Delta z_{i}$ is the associated thickness of layer $i$. $k$ is computed at each $z$-level (also called 'node'), either as the mean between values computed at two neighbouring layers or as the one-sided value at the upper and lower interfaces of the ice-snow column with the atmosphere and the ocean. At these interfaces, an energy balance is assumed (Eq. (9), assuming that $d z / d t=0$ ) in order to diagnose the temperatures at the extremities. This introduces half-distances in an otherwise uniform grid. These interfacial temperatures are used in the calculation of surface and basal conductive fluxes.

The time discretization of the heat equation is Euler-backward with two time levels ( $n$ and $n+1$ ), with $\Delta t$ the time step:

$\rho c^{\prime *} \frac{T_{i}^{*}-T_{i}^{n}}{\Delta t}=\rho \frac{E_{i}^{*}-E_{i}^{n}}{\Delta t}=\frac{\Delta}{\Delta z_{i}}\left(k \frac{\partial T^{*}}{\partial z}+R\right)$.

In this equation, we retain the continuous form of the conductive heat flux for simplicity; and the $\Delta$ operator is the discrete differencing operator. The superscript $*$ denotes the intermediate value, during the iterative procedure and before convergence and final adjustments that lead to the legitimate $n+1$ fields (explained below). Specifically, the specific heat capacity of BL99 for discrete problems ( $c^{\prime}$, see Eq. 3 ) is used, which allows for an exact conserva- tion of enthalpy. Eq. (10) leads to a simple tri-diagonal matrix problem.

At each time level, the full thermodynamic problem is split in three steps. First, a temperature profile is computed solving for Eq. (10). The solution of Eq. (10) is done iteratively because of the non-linear coefficients ( $c^{\prime}$ and $k$ ). However, because the BL99 discretization leads to a solver close to Newton's iterative method (i.e., the $c^{\prime}$ term and explicit first derivatives used in diagnosing the surface top temperature) -referred hereafter as a quasi-Newtonian solver-, a suitable convergence is achieved normally in 5-10 iterations. ${ }^{1}$ The boundary condition is typically of flux form (i.e., Neumann-type) but if the surface temperature goes above the local melting point, the temperature is capped and becomes a Dirichlettype boundary condition. The excess energy is then treated in the next step. For this first step, the conservation of enthalpy can be written for the whole column as

$\sum_{i} \rho \frac{E_{i}^{*}-E_{i}^{n}}{\Delta t} \Delta z_{i}^{n}=\left[k \frac{\partial T^{*}}{\partial z}+R\right]_{b}^{s u}$

The second step is the computation of thickness changes at each interface, from the difference between the internal conductive and the net surface heat fluxes (Eq. (9)), i.e., the excess energy. The conductive fluxes are computed from the newly calculated $T_{i}^{*}$ profile. The coordinates of the upper and lower interfaces $\left(z_{s u}\right.$ and $\left.z_{b}\right)$ move, and the conservation of enthalpy can now be written for the whole sea ice column as:

$\sum_{i} \rho \frac{E_{i}^{*}-E_{i}^{n}}{\Delta t} \Delta z_{i}^{n}+\rho E_{s u}^{*} \frac{z_{s u}^{n+1}-z_{s u}^{n}}{\Delta t}-\rho E_{b}^{*} \frac{z_{b}^{n+1}-z_{b}^{n}}{\Delta t}=[F+R]_{b}^{s u}$,

where $E_{s u}^{*}\left(E_{b}^{*}\right)$ is the enthalpy of the forming or melting ice at the surface (at the base). For melting, $E_{s u}^{*}$ and $z_{s u}^{n+1}-z_{s u}^{n}$ are actually derived by integrating through the top ice layers until the excess energy available for melting (RHS of Eq. (9)) is reached. In non-pathological cases, the integration does not need to be carried out further than the first top layer, which means $E_{s u}^{*}$ is most often the enthalpy of this layer. If the salinity of forming or melting bottom ice remains the same constant, as well as the bottom temperature remains fixed at freezing sea temperature, then $E_{b}^{*}$ remains a constant.

The third and last step is remapping of enthalpy. This step is required because following sea ice growth and melt, the vertical grid must be updated to adjust to the new thickness. To conserve enthalpy, the latter is equally redistributed on the new grid in a way that ensures the conservation of the total enthalpy $\left(\mathrm{J} \mathrm{m}^{-2}\right)$. Once this step is achieved, the enthalpies reach their final values at stage $n+1$. The conservation of enthalpy following this step reads:

$\sum_{i} \rho E_{i}^{n+1} \Delta z_{i}^{n+1}=\sum_{i} \rho E_{i}^{*} \Delta z_{i}^{n}+\rho E_{s u}^{*}\left(z_{s u}^{n+1}-z_{s u}^{n}\right)-\rho E_{b}^{*}\left(z_{b}^{n+1}-z_{b}^{n}\right)$,

so that

$\sum_{i} \rho\left(E_{i}^{n+1} \Delta z_{i}^{n+1}-E_{i}^{n} \Delta z_{i}^{n}\right)=[F+R]_{b}^{s u} \Delta t$

Since the term on the right hand side is the only energy input to the system, the energy budget is closed. The temperature of each layer can be retrieved by inverting the enthalpy expression (Eq. (2)) in preparation for the next time step.

\footnotetext{
${ }^{1}$ It should be mentioned that the quasi-Newtonian solver in BL99 has been replaced by a more direct Newton's iterative procedure by Turner et al. (2013) who introduced a Jacobian-Free Newton Krylov (JNFK) method in their more complex 1D ice thermodynamic model.
} 
A note about the remapping stage: it can be shown that if the remapping is done following a 'donor cell' approach (as it is in most cases and here specifically, see Vancoppenolle et al., 2012, Section 3.5.1), this part would be first order accurate with increased resolution.

\section{Equation for the Finite Volume (FV) model}

The discretized form of the heat equation for the $\sigma$-coordinate system is now presented for the case of the Finite Volume discretization. The presentation in HTB05 differs as they took advantage of some of the compaction offered in $\sigma$. Starting from the conservation of enthalpy in $\sigma$-coordinates (Eq. (8)), integrated between the two consecutive $\sigma$ surfaces defining layer $i$, assuming that the variables are constant inside these boundaries, the FV discretization leads for one layer to:

$\frac{\partial\left(\rho E_{i} \Delta z_{i}\right)}{\partial t}+\Delta(\rho E \omega)=\Delta\left(k \frac{\partial T}{\partial z}+R\right)$,

where $\Delta$ takes the difference of expressions evaluated on the top and bottom $\sigma$ surfaces of layer $i$. As $\Delta \sigma_{i}$ is constant between two $\sigma$ surfaces, it can be discarded and thus does not appear above. The conservation of enthalpy is not obvious as we are now dealing with a moving grid. Departing here from HTB05, two approximations are now introduced during the time-discretization. First, we separate the time derivative on the left-hand side into one tendency for energy and the other one for height:

$$
\begin{aligned}
& \rho \frac{E_{i}^{n+1}-E_{i}^{n}}{\Delta t} \Delta z_{i}^{n+1}+\rho E_{i}^{n} \frac{\Delta z_{i}^{n+1}-\Delta z_{i}^{n}}{\Delta t}+\Delta\left(\rho E^{n+1} \omega\right) \\
& =\Delta\left(k \frac{\partial T^{n+1}}{\partial z}+R\right),
\end{aligned}
$$

which still conserves enthalpy as one can see that the two time tendencies can simplify to $\rho\left(E^{n+1} \Delta z^{n+1}-E^{n} \Delta z^{n}\right) / \Delta t$ (due to the choice of time level for thickness and enthalpy that factor the two time tendency terms) Then, we can introduce the BL99 exact discrete differentiation of enthalpy (Eq. (3)) in order to get

$$
\begin{aligned}
& \rho c^{\prime} \frac{T_{i}^{n+1}-T_{i}^{n}}{\Delta t} \Delta z_{i}^{n+1}+\rho \Delta\left(c^{\prime}\left(T^{n+1}-T^{n}\right) w\right) \\
& =-\rho E_{i}^{n} \frac{\Delta z_{i}^{n+1}-\Delta z_{i}^{n}}{\Delta t}+\Delta\left(k \frac{\partial T^{n+1}}{\partial z}-\rho E^{n} w+R\right),
\end{aligned}
$$

where we seek a solution for $T^{n+1}$ by solving a simple tri-diagonal matrix problem. $\omega$ and $\Delta z^{n+1}$ will be discussed below. Since the problem is non-linear due to $c^{\prime}$ and $k$ depending on $T$, an iteration loop for time $n+1$ is required as for the FD method. It can then be shown that, by summing in the vertical direction

$$
\sum_{i} \rho\left(\frac{E_{i}^{n+1} \Delta z_{i}^{n+1}-E_{i}^{n} \Delta z_{i}^{n}}{\Delta t}\right)=\left[\left(k{\frac{\partial T^{n+1}}{\partial z}}^{n}-\rho \omega E^{n+1}+R\right)\right]_{b}^{s u},
$$

where the right-hand-side controls the amount of energy entering or leaving the domain. The boundary condition follows again Eq. (9) and is equivalent in our notation to

$k{\frac{\partial T^{n+1}}{\partial z}}^{n}-\omega \rho E^{n+1}=F$,

where $F$ is the net heat flux at the interface. This boundary condition is then used to diagnose $\omega$ at the interface and to derive the variation in $\Delta z$. Reintroducing this expression into Eq. (18) leads to the same expression derived in Eq. (14). The energy budget is therefore also closed for the FV method.

The original formulation of HTB05 is equivalent to rewriting Eq. (16) with the first two terms of the left hand side combined as
$\rho\left(E_{i}^{n+1} \Delta z_{i}^{n+1}-E_{i}^{n} \Delta z_{i}^{n}\right) / \Delta t$. Because $\Delta z^{n+1}$ and $\Delta z^{n}$ are not equal, the exact discretization of BL99 for enthalpy cannot be used. The authors therefore resorted to split the enthalpy between a quasi linear $c_{0}$ and non-linear $L_{0}$ parts (see Eq. (2)). The non-linear part is then moved to the right hand side and, after each tri-diagonal step, the temperature is iterated with an under-relaxation method (HTB05 method is otherwise enthalpy conserving too). One drawback, therefore, of the original HTB05 approach is the loss of the quasi-Newtonian characteristic which leads to poorer convergence (shown later). Retaining a quasi-Newtonian solver, as proposed here, is critical.

The advection is treated implicitly and with a first-order upwind scheme in HTB05. We found however that a centered implicit scheme has some advantages in terms of convergence with resolution while we did not find any stability or major dispersion problems associated with it. Also, HTB05 treats explicitly the ice-snow interface temperature as opposed to what is done in conventional implementations of BL99 where the interface temperature can be removed from the unknowns using the equality of the conductive heat flux on both sides of the interface. A priori, the explicit representation of the interface temperature presents the advantage of enforcing that the latter has to be below the lowest melting point of the two media. However in presence of thin snow, the temperature solver convergence is sometimes not achieved depending on the user-specified value of the minimum snow thickness possible. Hence, the physically based representation of HTB05 leads to a poorer numerically-posed problem, and therefore we decided to follow the conventional implementation of BL99 in this matter.

\section{Equation for the Finite Element (FE) model}

Finally, the discretized form of the heat equation for the $\sigma$-coordinate system using Finite Elements is derived. The Finite Element formulation departs from the FV formulation as it starts from Eq. (5), and in that it applies the Galerkin method in a weak sense

$$
\int_{0}^{1} \rho\left(\frac{\partial E}{\partial t}+\dot{\sigma} \frac{\partial E}{\partial \sigma}\right) \frac{\partial z}{\partial \sigma} \phi_{i}(\sigma) d \sigma=\int_{0}^{1} \frac{\partial}{\partial z}\left(K \frac{\partial T}{\partial z}+R\right) \frac{\partial z}{\partial \sigma} \phi_{i}(\sigma) d \sigma,
$$

where $\phi_{i}$ forms a basis of test functions and the reference to the time derivative being done for constant $\sigma$ is dropped. For simplicity, the basis $\left(\phi_{i}(\sigma)\right)_{i=1 N}$ is taken as the series of piecewise linear "hat" functions ( $C^{0}$ continuous) discretizing the ice-snow compound into $N-1$ contiguous layers (or elements) and $N$ vertices (or nodes). Hence, the discretization offers at best second order convergence. The advective term is then rewritten in terms of $z$ for simplicity, and all integrations are done in terms of $z$.

$\int_{z_{b}}^{z_{s u}} \rho \frac{\partial E}{\partial t} \phi_{i} d z+\int_{z_{b}}^{z_{s u}} \rho \omega \frac{\partial E}{\partial z} \phi_{i} d z=\int_{z_{b}}^{z_{s u}} \frac{\partial}{\partial z}\left(K \frac{\partial T}{\partial z}+R\right) \phi_{i} d z$,

where we introduced again $\omega=\dot{\sigma}(\partial z / \partial s)$. Typically, for the "weak form", the advection and heat conduction are integrated by parts, introducing a boundary condition to the problem:

$$
\begin{gathered}
\int_{z_{b}}^{z_{s u}} \rho \frac{\partial E}{\partial t} \phi_{i} d z-\int_{z_{b}}^{z_{s u}} E \frac{\partial\left(\rho \phi_{i} \omega\right)}{\partial z} d z+\int_{z_{b}}^{z_{s u}}\left[K \frac{\partial T}{\partial z}+R\right] \frac{\partial \phi_{i}}{\partial z} d z \\
=\left[\left(K \frac{\partial T}{\partial z}-\omega \rho E+R\right) \phi_{i}\right]_{b}^{s u} .
\end{gathered}
$$

A few approximations are then necessary at this stage. First, the coefficients, such as density and thermal conductivity, need to be treated as piecewise constants inside each element and receive therefore the $e$ subscript. Second, the energy in the advective integral is also treated as piecewise constant. This leads to 


$$
\begin{aligned}
& \int_{z_{b}}^{z_{s u}} \rho_{e} \frac{\partial E}{\partial t} \phi_{i} d z-\int_{z_{b}}^{z_{s u}} \rho_{e} E_{e} \frac{\partial \phi_{i} \omega}{\partial z} d z+\int_{z_{b}}^{z_{s u}}\left[K_{e} \frac{\partial T}{\partial z}+R\right] \frac{\partial \phi_{i}}{\partial z} d z \\
& \simeq\left[\left(K \frac{\partial T}{\partial z}-\omega \rho E+R\right) \phi_{i}\right]_{b}^{s u} .
\end{aligned}
$$

Third, the time tendency of the enthalpy is discretized using again an Euler-Backward formulation and rewritten in terms of the time tendency of temperature times the BL99 heat capacity (also defined as element-wise value)

$$
\begin{aligned}
& \int_{z_{b}}^{z_{s u}} \rho_{e} c_{e}^{\prime} \frac{T^{n+1}-T^{n}}{\Delta t} \phi_{i} d z-\int_{z_{b}}^{z_{s u}} \rho_{e} E_{e} \frac{\partial \phi_{i} \omega}{\partial z} d z \\
& +\int_{z_{b}}^{z_{s u}}\left[K_{e} \frac{\partial T^{n+1}}{\partial z}+R\right] \frac{\partial \phi_{i}}{\partial z} d z \simeq\left[\left(K{\frac{\partial T^{n+1}}{\partial z}}^{n}-\omega \rho E+R\right) \phi_{i}\right]_{b}^{s u} .
\end{aligned}
$$

Fourth, the first integral in Eq. (24) is evaluated at $t=t^{n+1}$ as far as the boundaries of the integral are concerned. This eliminates the complication of the moving $b$ and su levels during the time integration. The enthalpy in the second integral is assumed at $t=t^{n}$. The final equation is then

$$
\begin{aligned}
& \int_{z_{b}^{n+1}}^{z_{s u}^{n+1}} \rho_{e} c_{e}^{\prime} \frac{T^{n+1}-T^{n}}{\Delta t} \phi_{i} d z-\int_{z_{b}}^{z_{s u}} \rho_{e} E_{e}^{n} \frac{\partial \phi_{i} \omega}{\partial z} d z \\
& +\int_{z_{b}}^{z_{s u}}\left[K_{e} \frac{\partial T^{n+1}}{\partial z}+R\right] \frac{\partial \phi_{i}}{\partial z} d z \simeq\left[\left(K \frac{\partial T^{n+1}}{\partial z}-\omega \rho E^{n+1}+R\right) \phi_{i}\right]_{b}^{s u}
\end{aligned}
$$

where we seek a solution for $T$ in the space spanned by $\left(\phi_{i}(s)\right)_{i=1, N}$ as $\widetilde{T}=\sum_{j} T_{j} \phi_{j}$ by solving for each test function in the basis. This again leads to a simple tri-diagonal problem. $\omega$ (which is linear by definition) is also discretized using the same basis and the internal transport term becomes part of the right hand side. The conservation of enthalpy can then be proven using the test function $\phi=1$ and taking advantage of $T_{e}=1 / h_{e} \int^{e} T d z$ that defines the element-wise temperature and therefore enthalpy ( $h_{e}$ is the thickness of one element). In this case, the discretized enthalpy equation collapses to

$$
\sum_{e} \rho_{e}\left[\left(\frac{E_{e}^{n+1}-E_{e}^{n}}{\Delta t}\right) h_{e}^{n+1}-E_{e}^{n} \Delta_{e} \omega\right]=\left[\left(K \frac{\partial T^{n+1}}{\partial z}-\omega \rho E^{n+1}+R\right)\right]_{b}^{s u},
$$

where the $\Delta_{e}$ function defines the difference between the top and bottom surface of each layer. However, $-\Delta_{e} \omega$ is exactly defined as $\left(h_{e}^{n+1}-h_{e}^{n}\right) / \Delta t$, therefore the $E_{e}^{n} h_{e}^{n+1}$ terms cancel out leaving

$\sum_{e} \rho_{e} \frac{E_{e}^{n+1} h_{e}^{n+1}-E_{e}^{n} h_{e}^{n}}{\Delta t}=\left[\left(K \frac{\partial T^{n+1}}{\partial z}-\omega \rho E^{n+1}+R\right)\right]_{b}^{s u}$.

Because the boundary condition (Eq. (9)) is equivalent in our notation to

$\left[K{\frac{\partial T^{n+1}}{\partial z}}^{n}-\omega \rho E^{n+1}\right]_{b}^{s u}=F_{s}-F_{b}$

the time tendency of the total enthalpy of the snow-ice system is only dependent on the energy input. Thus, the energy budget is completely closed and leads to an equivalent expression as Eq. (14).

Again, Eq. (25) is solved iteratively as a quasi-Newtonian solver. And, as for the FV method, we concomitantly iterate on the volume transport $\omega$. As is, the treatment of the advection term is secondorder accurate with resolution.

\section{Intercomparison in the framework of the SHEBA experiment}

The SIMIP2 protocol is used for this comparison, keeping in line with HTB05. It is based on measurements made during the SHEBA experiment (Persson et al., 2002). This experiment was an international effort to increase the understanding of sea-ice growth and decay, and interactions and exchanges with the atmosphere and ocean (Perovich et al., 1999).

Ice Station SHEBA consisted of several observational sites around the main camp (ice breaker) drifting westward with the Beaufort Gyre. Hourly measurements of atmospheric variables, turbulent heat exchange, albedo (Curry et al., 2001), oceanic heat flux (McPhee et al., 2005), temperature profiles obtained from thermistor chains through the snow-ice system and upper ocean, and thickness of ice and snow, among other variables, were performed, starting in fall 1997 and ending at the beginning of the next fall, covering almost a full seasonal cycle continuously. Measurements of the vertical position of the air/snow and snow/ice interfaces were made visually each day from ablation stakes (Perovich and Elder, 2001). The position of the basal ice was taken manually from a thickness gauge which is unfrozen (short heat pulse in wire) and pulled up until a plate at the end of the gauge hits the bottom ice and height is read from the gauge. Positions can also be inferred from the change in gradients of thermistorderived temperature profiles (Huwald et al., 2005b).

While different sites were chosen for the ice mass balance observations, we focused here on the Pittsburgh site as in HTB05. This site was located on level multi-year ice, and thus provides a set of measurements that are most consistent with 1D thermodynamic models.

The temperature data from the Pittsburgh site (Fig. 1) displays a basal build-up of the ice during the winter period with low temperature at the ice-snow surface of $-24{ }^{\circ} \mathrm{C}$ in three cold episodes (late December-early January, mid-January and mid-February). The maximum ice thickness recorded was $2.6 \mathrm{~m}$ in mid-May from an initial $1.9 \mathrm{~m}$ in the fall. Basal melt started in late May and was still going on (but very weakly) when the measurements stopped in September 25 ( $40 \mathrm{~cm}$ basal loss overall). At the surface, the $20 \mathrm{~cm}$ snow cover melted quickly in late May and left the ice bare by June 14 . Then, the surface ablation removed an additional $80 \mathrm{~cm}$ of ice in less than two months.

The three models (FD, FV, FE) were initialized with a linear temperature profile in the snow and the observed temperature profile in the ice from the SHEBA/Pittsburgh data. As already mentioned in the introduction, the salinity is here considered constant and uniform at $4 \mathrm{~g} / \mathrm{kg}$, since our goal is not to reproduce in detail the observed fields, but rather to define a simpler model background

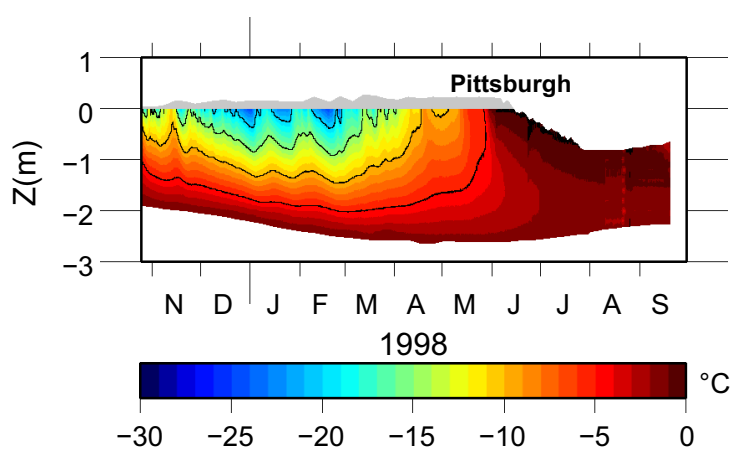

Fig. 1. SHEBA temperature data plotted following the accretion/ablation rate of the surface and base of ice for the Pittsburgh Station. Temperature contours are plotted every $5^{\circ} \mathrm{C}$. 
state to which we can compare different numerical approaches to the heat equation problem.

The short and long wave radiation, wind speed, air temperature, specific humidity, precipitation, surface albedo and oceanic heat flux at the bottom of the ice are taken from SHEBA measurements and are used to force at the surface the different models using the same bulk formulae provided in LIM3. The time-step is fixed to one hour in all models and for all resolutions.

All three models were proof-checked for energy conservation errors. In all models, the most tedious parts were the treatment of the disappearing snow and the possibility for the temperature solver to oscillate between surface melt and refreezing. The thermodynamic solver in all three models is said to have converged when the maximum temperature increment falls below $10^{-12}{ }^{\circ} \mathrm{C}$ in less than 50 iterations.

Fig. 2 illustrates the convergence of the temperature solver (energy error and iteration count at bailout) of the present FV formulation relative to the original HTB05 model. While the original HTB05 requires already a large number of iterations to converge in the relative quiescent period of ice formation, its convergence quickly deteriorates by another order of magnitude during the more numerically challenging surface melt period (with several bailouts before final convergence could be reached with a criteria of a maximum temperature increment of $10^{-12}{ }^{\circ} \mathrm{C}$ or a maximum iteration count of 20,000). Conversely, the FV formulation, with its quasi-Newtonian solver, only experienced a doubling of the iteration count to reach the requested level of energy error. Hence, the convergence of the HTB05 model has been improved by a factor of 10 during the cold season in the absence of surface melting and sometimes more than a factor of 100 during periods of surface melting. This is a striking evidence for the need of a solver with the basic convergence properties closest to Newton's method.

Fig. 3 shows the timeseries of ice thickness, iteration count to reach full convergence in the temperature solver for all three models, with resolutions of 3, 20 and 100 layers of ice respectively, with 1 snow layer for the first case and 5 snow layers otherwise. In terms of thickness, the FV and FD solutions are in general indistinguishable. The FE model is at low resolution clearly apart from the two others, but in fact all models converge with increasing resolu-
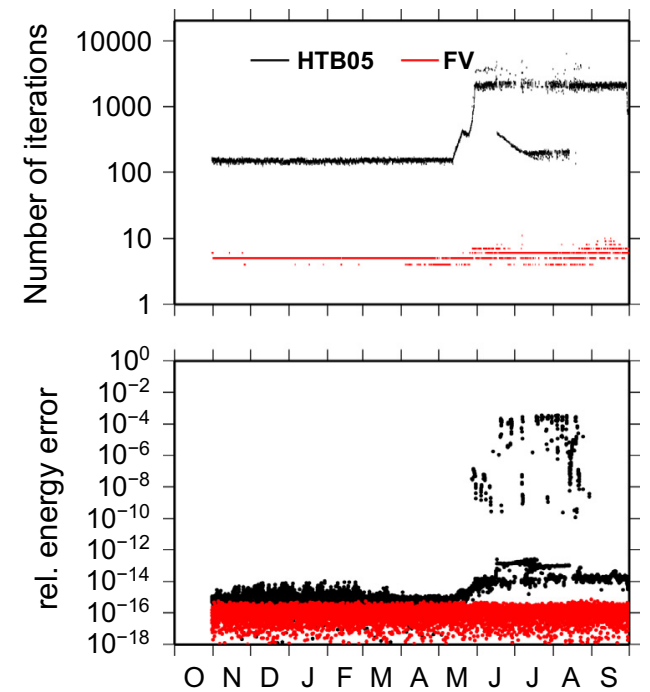

Fig. 2. Iteration count and relative energy error at convergence (or at bail-out in case of convergence failure) for a resolution of one snow and 10 ice layers for the old FV formulation of HTB05 (black) and the present (red). The melting period starting from May 1998 is clearly visible as a time when the convergence is harder to achieve. (For interpretation of the references to colour in this figure caption, the reader is referred to the web version of this article.) tion to the point that the discrepancy is virtually not discernible at 100 ice layers. The FD and FV solutions are, with 3 vertical ice layers, already within $3 \mathrm{~cm}$ of the 200 ice layer solution, whereas the FE solution, at the same resolution, stands out with a $9 \mathrm{~cm}$ difference. The FD method has in general the best convergence history of the temperature solver when compared to FV and FE, as the latter methods are slightly penalized by the presence of extra terms in the temperature equation. In the FV case, this means that the model only needs one more iteration occasionally ( 0.2 iterations per time-step at 20 ice layers on averaged, 0.6 at 100 ice layers). The FE solver is more penalized with an additional 3 iterations per time-step, independent of the season or the resolution in the ice. Increasing the vertical resolution in the ice degrades only very slightly the convergence performance for all models.

Table 1 shows the RMS error for different resolutions and gives the effective convergence order with resolution for each model, and for different seasons (see also Fig. 4). All three models were run in a 200 ice layer configuration. These respective solutions are considered the best guess "truth" for each model, and are taken as references for the comparisons and benchmarking. The largest errors are observed in summer (April to September) during the surface melt period, when the interface experiences the fastest rate of change. The summer errors are often an order of magnitude larger than those during winter (November to March), leading the overall error for the full annual period. The FE model shows the lowest errors in all seasons and also the best error convergence with vertical resolution. The winter error convergence is very dependent on the overall numerical order of the discretization and a centered advection scheme is then beneficial as the FE and FV examples demonstrate that a second order of accuracy can be achieved. However, the summer accuracy rates are all close to first order and this seems to be related to the top surface treatment in all models. During surface melt, the surface treatment requires an enthalpy estimate, and by definition, the enthalpy of a melting surface is zero which would lead to an infinite rate of ablation. In such a case, the FV/FD models off-center the problem by applying the enthalpy of the closest existing layer, which re-introduces a firstorder approximation. The FE discretization is by nature first-order at the boundary (off-centered), although the boundary treatment does not require an explicit definition of surface enthalpy. Hence, all models tend to fall back to first order during the melt season.

The internal temperature field is plotted in Fig. 5 for each formulation (FD, FV, FE) at three resolutions $(3 / 1,20 / 5$ and $100 / 5$ ice/snow layers) as a function of vertical position and time. The vertical $z$-coordinate follows the simulated rate of surface ablation. The mean of the three models at the highest resolution (200/5 ice/ snow layers) is considered as the "best guess" solution. The term bias hereafter refers to the difference of any solution relative to this "best guess" solution. The most striking feature seen in Fig. 5 is the $0.3-0.5{ }^{\circ} \mathrm{C}$ warm bias in the first layer of all models at the lowest resolution (three ice layers). Nonetheless, the bias decreases as the resolution is increased for all three numerical formulations. This is expected as these seek the solution of the same problem. The second striking aspect in Fig. 5 are the diurnal spikes. At three ice layers, the diurnal spikes of surface temperature penetrate deeper in the ice since the layers are thicker. As the thickness of the layers decreases with increasing vertical resolution, the diurnal spikes are attenuated and much shallower as well. It is interesting to note that diurnal sub-surface oscillations in ice temperature are seen in the SHEBA data. However, whether the simulated diurnal oscillations are of numerical nature, due to the lack of resolution, or rather a realistic feature is hard to decide. This is because observational errors are the largest in this period, due to solar heating of the white rods where the thermistors reside (Don Perovich, pers. comm., 2012). In this regard, we note that the FV/FD models have a natural resolution enhancement at the surface since a layer-centered 

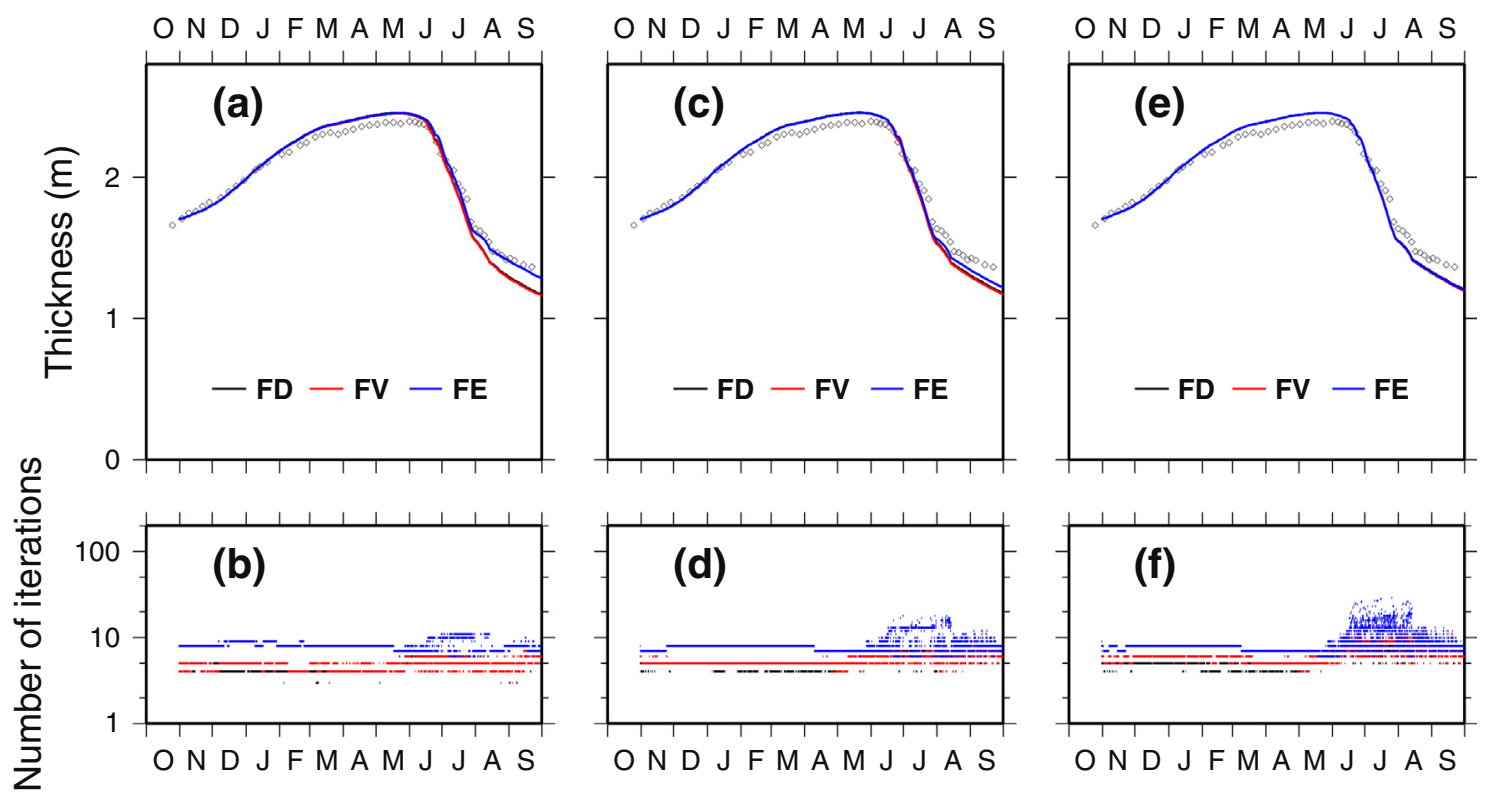

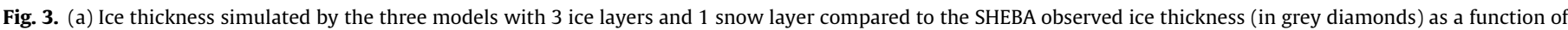

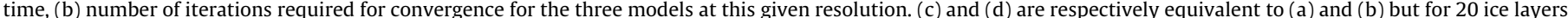

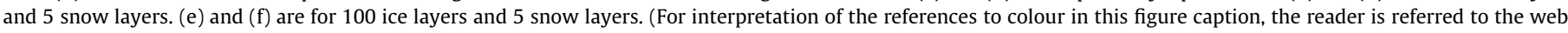
version of this article.)

Table 1

RMS errors for each model in the ice column, as a function of resolution (3 to 100 layers) and three periods (full simulation, winter and summer) in temperature $\left({ }^{\circ} \mathrm{C}\right.$ ). The reference in the error calculation is the highest resolution (200 layers) run for each particular model. The snow resolution is 1 layer for the 3 ice layer case and 5 otherwise. The averaged order of spatial convergence is computed for each model and is shown in the last column.

\begin{tabular}{lllllll}
\hline Models & 3 & 10 & 20 & 50 & 100 & Order \\
\hline \multicolumn{2}{l}{ Full period } & & & & & \\
FD & 1.981 & $2.97310^{-1}$ & $1.91510^{-1}$ & $6.55010^{-2}$ & $2.20910^{-2}$ & 1.24 \\
FV & 1.979 & $2.62910^{-1}$ & $1.45410^{-1}$ & $5.68310^{-2}$ & $2.02810^{-2}$ & 1.26 \\
FE & 3.970 & $1.33410^{-1}$ & $6.84010^{-2}$ & $2.77110^{-2}$ & $9.54410^{-3}$ & 1.58 \\
Winter & & & & & & \\
FD & 2.586 & $1.48510^{-1}$ & $7.01110^{-2}$ & $2.49810^{-2}$ & $8.60610^{-3}$ & 1.52 \\
FV & 2.605 & $9.43610^{-2}$ & $2.44010^{-2}$ & $3.86610^{-3}$ & $8.26210^{-4}$ & 2.24 \\
FE & 5.314 & $5.71510^{-2}$ & $1.43710^{-2}$ & $2.45910^{-3}$ & $5.71510^{-4}$ & 2.45 \\
Summer & & & & & & \\
FD & 1.276 & $3.78810^{-1}$ & $2.51210^{-1}$ & $8.56710^{-2}$ & $2.88510^{-2}$ & 1.08 \\
FV & 1.235 & $3.45210^{-1}$ & $1.95610^{-1}$ & $7.68410^{-2}$ & $2.74310^{-2}$ & 1.10 \\
FE & 2.318 & $1.72910^{-1}$ & $9.16410^{-2}$ & $3.74310^{-2}$ & $1.29110^{-2}$ & 1.40 \\
\hline
\end{tabular}

temperature is adjacent to the top surface temperature, effectively doubling the resolution relative to the FE model.

Since most of the error appears to be due to the surface processes, we focus now on the surface temperature of the ice/snow medium (Fig. 6) and investigate in more details the time-evolution of the error (again relative to the "reference" 200 ice layer solution) for different vertical resolutions of the FV model. The low resolution (three and 10 ice layers) solutions exhibit strong swings related to diurnal cycles. The error is particularly large during the melt season (starting in June) when episodic refreeze events happen (maximum of $4{ }^{\circ} \mathrm{C}$ error for three ice and one snow layers). With 10 ice and five snow layers, the error is reduced by a factor of two in summer and even more in winter. And the same is also true between 10 and 20 ice layers. However, from a large scale climate modelling perspective, the error magnitude may appear trivial: $0.2^{\circ} \mathrm{C}$ in bias and $0.6^{\circ} \mathrm{C}$ in RMS error from May 1,1998 to September 1, 1998 for three ice and one snow layer. Note that

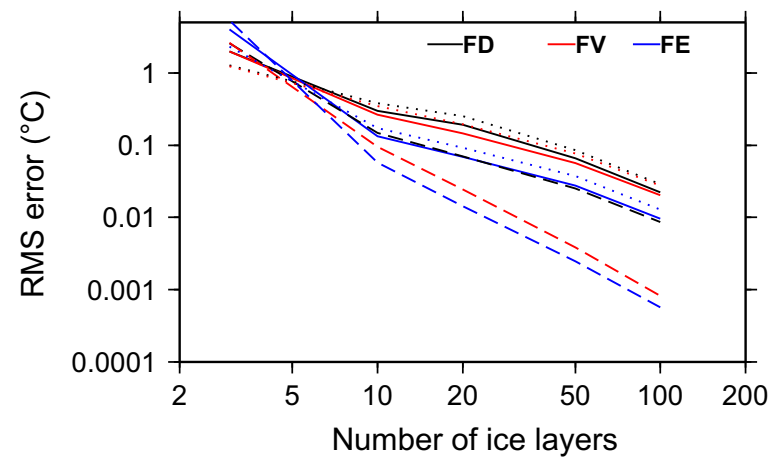

Fig. 4. Convergence with resolution (number of ice layers) for the three models, black for FD, blue for FE and red for FV. The solid lines are for the entire period, the dashed lines are for winter and dotted for summer. The reference solution for each model is considered to be the solution with highest resolution, i.e., 200 ice layers, for that particular model. (For interpretation of the references to colour in this figure caption, the reader is referred to the web version of this article.)

the convergence in RMS error is again only first order with resolution during this period.

We also compare the different numerical approaches in terms of pure clock time performance. The scores in Table 2 show that, once each code is stripped of $\mathrm{I} / \mathrm{O}$ and fully rerun for the entire period on a Ubuntu 10.432 bits with a Intel Core2 CPU T5500 nominally clocked at $1.66 \mathrm{GHz}$, the cost increase with resolution is less than linear until the count of 50 ice layers for the FD model is reached (not shown), and slightly later for the other two models (FE and FV). The FD model is slower by $20 \%$ relative to the two other models at low resolution. The difference keeps increasing with resolution though and reaches a factor 5 at 200 ice and 5 snow layers. This additional cost of FD originates from the vertical remapping as the thermodynamic solver shows relatively similar performances. Remapping is indeed costly as some double nested loops are involved. One reviewer justly noted that the remapping algorithm can be better optimized for large numbers of layers. The modification is relatively simple and avoids the double nested 


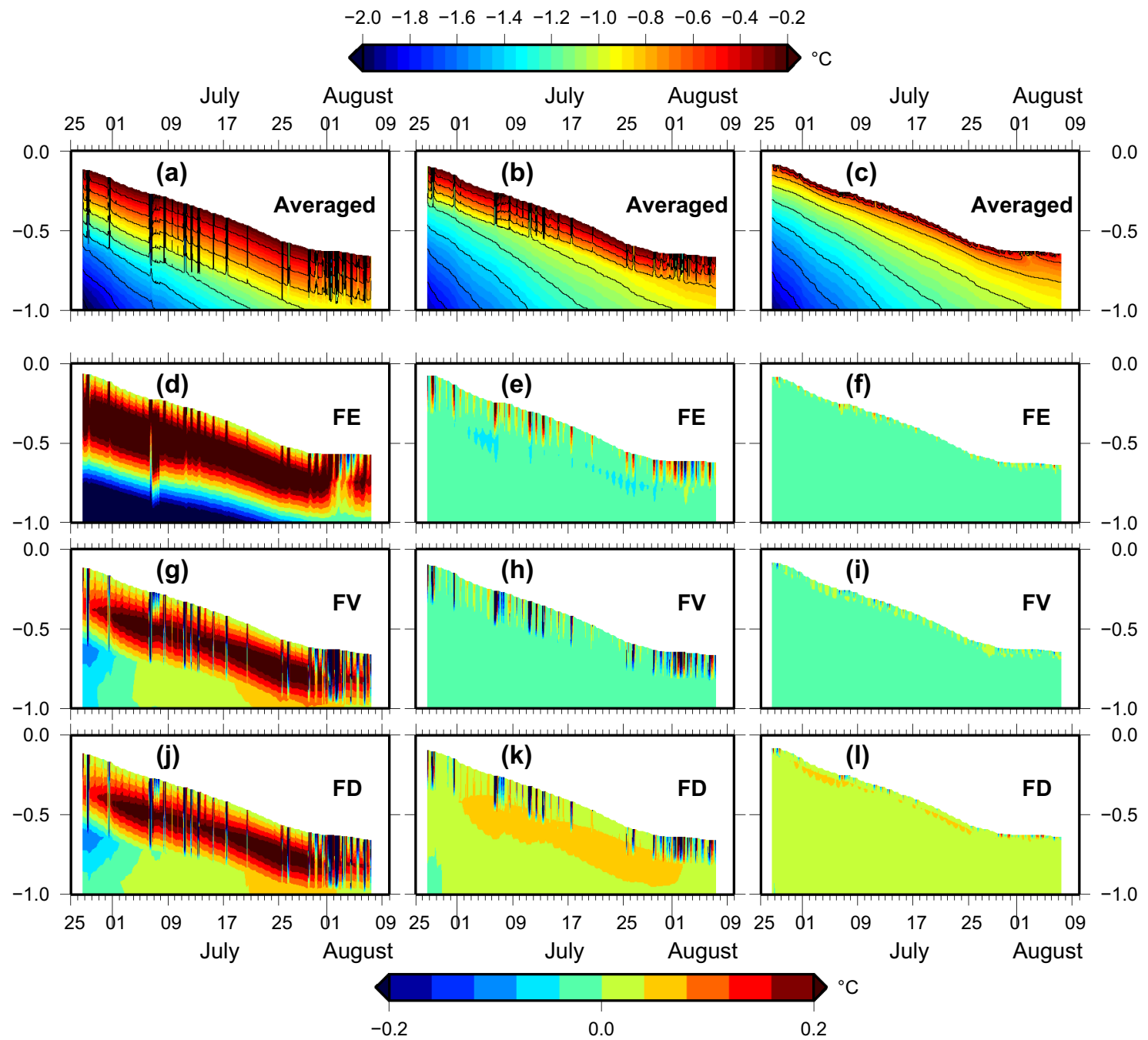

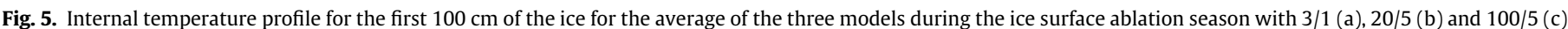

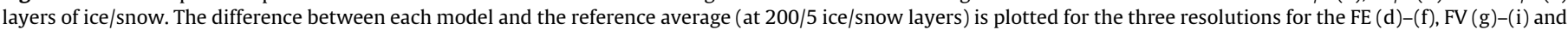
FD (j)-(l) models, each series of panels following respectively the resolution used in (a)-(c) and with vertical coordinate representing ablation.

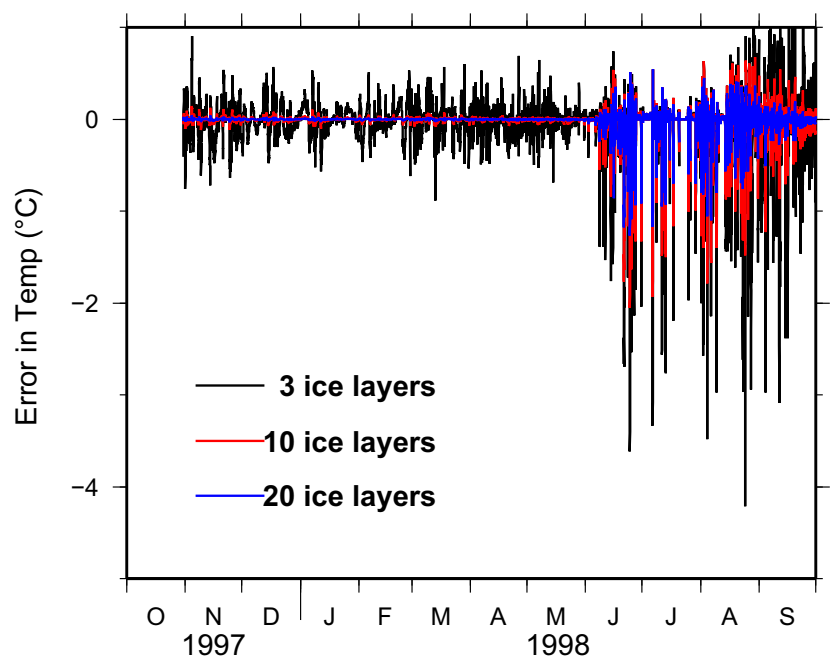

Fig. 6. Surface temperature over the snow/ice for the three different resolutions of the FV model: (black) 3 ice and 1 snow layers, (red) 10 ice and 5 snow layers, (blue) 20 ice and 5 snow layers. (For interpretation of the references to colour in this figure caption, the reader is referred to the web version of this article.)
Table 2

CPU time performances (in s) for the whole simulation (8208 time-steps) for the FD, FV and FE models as a function of vertical resolution $(3+1$ corresponds to three ice and one snow layers; $20+5$ to 20 ice and 5 snow layers, etc...).

\begin{tabular}{lllll}
\hline Models & $3+1$ & $20+5$ & $100+5$ & $200+5$ \\
\hline FD & 1.40 & 2.00 & 9.72 & 31.00 \\
FV & 1.19 & 1.61 & 3.56 & 6.14 \\
FE & 1.22 & 1.59 & 3.23 & 5.38 \\
\hline
\end{tabular}

loops, to the point that the additional cost of the remapping can decrease to only $5 \%$ relative to $\mathrm{FV}$. That increasing the resolution does not degrade linearly the time performances of FV and FE (below 100 ice layers) is likely related to the fact that the problem is small enough to reside entirely in the cache of the computer processor (memory access is a classical bottle-neck in numerical climate models). As to illustrate the simplicity of the FV method, the programming complexity of the FV formulation is limited to the addition of a few extra terms in the heat equation, while avoiding a remapping algorithm.

Another important numerical aspect is the behaviour of the models for situations where the ice layer is getting thin and 

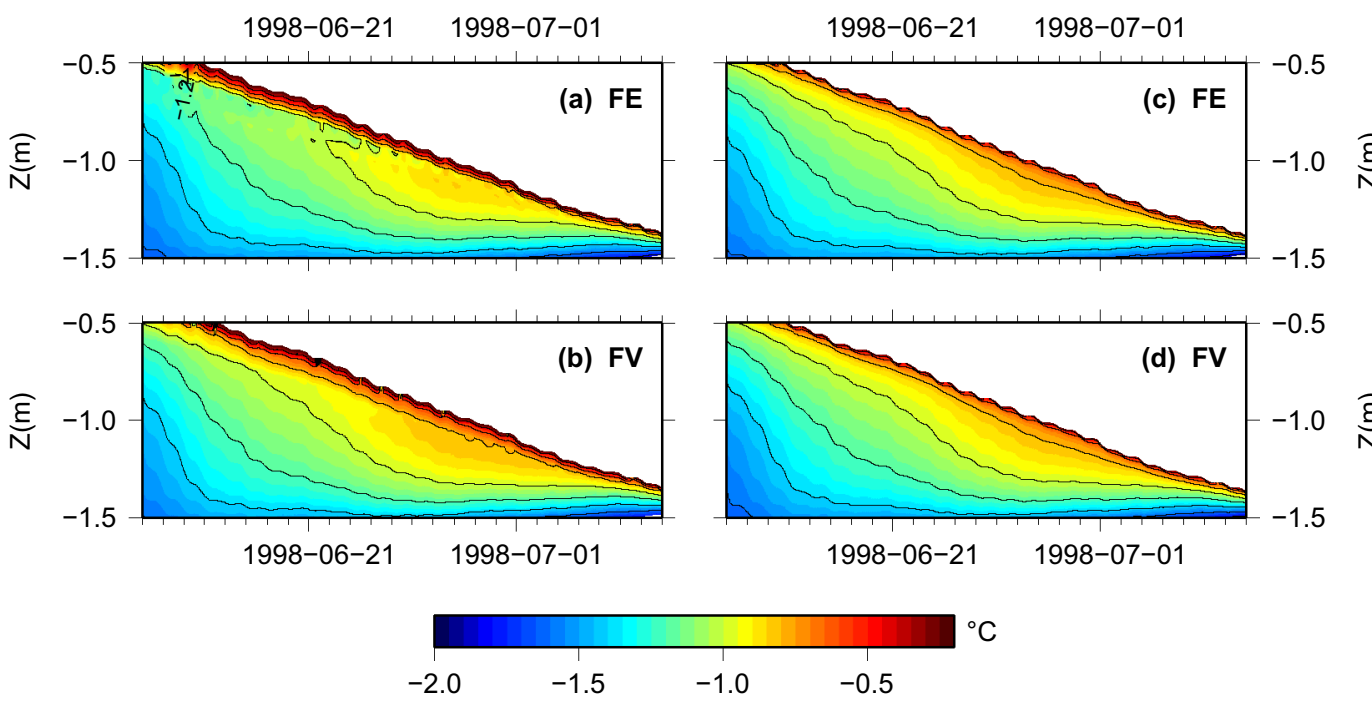

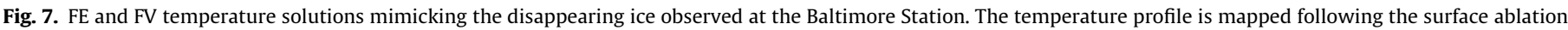
rate. The two top panels (a) and (c) represent the FE solution with 10 (left) and 100 (right) ice layers. The two bottom panels (b) and (d) are the equivalent for FV.

eventually disappears. Such is the case for instance at the SHEBA site Baltimore where the ice during summer was heavily ponded. We are not truly interested in reproducing the complex processes occurring at the Baltimore site, which is again beyond the scope of this paper, but we can use some of the information available at this site for setting up an equivalent modelling case with thin and disappearing ice. For partially reproducing the large rate in surface melt, the albedo derived for the Pittsburgh site was lowered by $20 \%$, the atmospheric forcing being otherwise unmodified. The FE and FV models were initialized with a linear temperature profile, no snow and $0.4 \mathrm{~m}$ of ice thickness at the same date as for the Pittsburgh station for two different resolutions: 10 or 100 layers of ice and only one layer of snow. The FE model tends to develop counter temperature gradients just below the surface for the low resolution case (Fig. 7) while the FV model does not show such features. At higher resolution, FE no longer displays counter gradients and the two model results are very similar. Hence, the counter gradient at low resolution in the FE model appears to be a numerical artifact.

The problem is not investigated further but may be related to the different grid staggering in the FV and FE models, i.e. the fact that the prognostic temperature is located at the nodes, the interfaces of each computational layer in the FE context, while the enthalpy is based on the centered-layer temperature (the mean of the two interface temperatures). This allows for a free numerical mode in the form of oscillations at the nodes while the overall enthalpy is still conserved. These oscillations are likely exacerbated by large heat fluxes and lack of resolution of the surface boundary layer.

In the FV and FE sigma models, additional limit cycles (endless oscillations between two temperature approximate solutions) were found for thin ice and very high number of layers, which means that the sigma approach is no longer viable when ice thickness falls below $1 \mathrm{~cm}$. Note that the FD model also suffers from convergence issues for moderately thin ice that were related to the internal snow temperature reaching melting point. Hence, none of the 1D thermodynamic models presented here is truly viable for thin ice and high number of ice layers. A degraded resolution method appears therefore desirable to treat the disappearing ice case. In terms of resolution this means something comparable to the default value of layers suggested in CICE or LIM3, which is typically 3-4 for the ice. Arguably, disappearing ice can be even treated with one layer (linear profile), and to some degree, disappearing ice can be considered an academic problem, as in the ocean, the action of winds and waves tends to pile or raft thin ice into thicker ice chunks. The disappearing ice problem becomes then more of a lateral melting problem than a vertical one.

\section{Discussions and conclusions}

The vertical 1D sea-ice thermodynamic problem has been revisited and three different numerical model approaches have been compared using the SIMIP2 protocol, which is based on SHEBA observations. The investigated numerical methods include Finite Differences (FD) based on BL99, Finite Volumes (FV) based on HTB05, and Finite Elements (FE) also based on the vertical sigma coordinate approach of HTB05. All three methods are rigorously tested for strict enthalpy conservation. The FD implementation artificially separates temperature and changes in the vertical position in three distinct steps (the non-linear iterative vertical temperature solver followed by a thickness adjustment and a remapping step). The sigma-level transformation introduced by HTB05 leads to an elegant formulation where temperature and changes to the vertical position of the ice are solved together iteratively inside the vertical temperature solver. The remapping is no longer necessary as it is replaced by a vertical (grid-) advection term. This formulation is tested in the FV and FE frameworks. For the FV approach, a small modification to the original HTB05 formulation allows for a large improvement in convergence of the nonlinear temperature solver, which makes the scheme affordable for climate models. In practice, the differences between the three formulations are small but most obvious during the melting season, while in terms of pure performance (clock time), the FV and FE models are superior to FD for large number of vertical ice layers. One reviewer suggested an improvement to the FD remapping step that considerably speeds up the time execution of the FD model, which is then only marginally more expensive than FV. The winter FD spatial convergence also shows that there may be an interest in increasing the order of the remapping technique, to at least second order.

The general question of the optimal number of ice layers is related to the processes that one would like to resolve. Obviously, if one is only interested in the large scale thermodynamic/dynamic response of ice, climate models will still be fine with 3-4 ice layers 
as this only reduces the thickness accuracy by a few centimeters. On the other hand, as interests in halo-dynamics and bio-physical coupling increase, 10 ice layers might be thought to be a minimum value that enables a fair representation of the vertical gradients present in the ice (Turner et al., 2013). In particular, according to Fig. 5 the brine fraction is expected to vary by $20 \%$ close to the ice surface between 3, 10 and more layers. The downside of increasing the vertical resolution is the increasing risk of violating an internal limitation of the model for thin ice, which we argue would then need to be addressed by a specific resolution degradation procedure.

The FE model shows very good order of accuracy. However, for moderately thick ice, melting condition and coarse resolution, it generates counter-gradients in the temperature profile, while still conserving enthalpy. Because of this limitation, the more reliable FV and FD formulations are recommended for accurate representation of sea-ice thermodynamics in large-scale climate models. FV is more cost-efficient at high number of layers than FD but a small modification to the remapping step in FD can help close the gap.

Recent efforts towards the improvement of the representation of sea ice halo-thermodynamics, notably accounting for sea ice desalination (Vancoppenolle et al., 2010; Griewank and Notz, 2013; Turner et al., 2013) and the "mushy layer" physics (Turner et al., 2013) are still based on a similar heat equation and on the same remapping technique as in BL99 and should benefit from the numerical suggestions made in this paper.

\section{Acknowledgements}

We are very grateful to Jean-Francois Lemieux from EC for his helpful suggestions on the first draft of the manuscript and to the two reviewers who greatly contributed to the improvement of the manuscript through their constructive comments. One reviewer, William Lipscomb, contributed to the improved remapping algorithm, which can be found as an electronic supplement to this manuscript.

\section{Appendix A. Supplementary material}

Supplementary data associated with this article can be found, in the online version, at http://dx.doi.org/10.1016/j.ocemod.2014.12. 006.

\section{References}

Bitz, C.M., Lipscomb, W.H., 1999. An energy-conservating thermodynamic model of sea ice. J. Geophys. Res. 104, 15669-15677.

Curry, J.A., Schramm, J.L., Perovich, D.K., Pinto, J.O., 2001. Applications of SHEBA/ FIRE data to evaluation of snow/ice albedo parameterizations. J. Geophys. Res. $106,15345-15355$

Ebert, E.E., Curry, J.A., 1993. An intermediate one-dimensional thermodynamic sea ice model for investigating ice-atmosphere interactions. J. Geophys. Res. 98 10085-10109.
Griewank, P.J., Notz, D., 2013. Insights into brine dynamics and sea ice desalination from a 1-D model study of gravity drainage. J. Geophys. Res. 118, 3370-3386.

Hunke, E.C., Lipscomb, W.H., 2010. CICE: the Los Alamos sea ice model documentation and software user's manual. Tech. rep., Los Alamos National Laboratory.

Hunke, E.C., Notz, D.K., Turner, A., Vancoppenolle, M., 2011. The multi-phase physics of sea ice: a review for modellers. Cryosphere 5, 989-1009.

Huwald, H., Tremblay, L.-B., Blatter, H., 2005a. A multilayer sigma-coordinate thermodynamic sea ice model: validation against surface heat budget of the Arctic Ocean (SHEBA)/ sea ice model intercomparison project part 2 (SIMIP2) data. J. Geophys. Res. 110, C05010.

Huwald, H., Tremblay, L.-B., Blatter, H., 2005b. Reconciling different observational data sets from surface heat budget of the Arctic Ocean (SHEBA) for model validation purposes. J. Geophys. Res. 110, C05009.

IOC, SCOR, IAPSO, 2010. The international thermodynamic equation of seawater 2010: calculation and use of thermodynamic properties. Intergovernmental Oceanographic Commission, Manuals and Guides, No. 56, UNESCO.

Kreyscher, M., Harder, M., Lemke, P., Flato, G.M., 2000. Results of the sea ice model intercomparison project: evaluation of sea ice rheology schemes for use in climate simulations. J. Geophys. Res. 105, 11299-11320.

Malmgren, F., 1927. On the properties of sea ice. In: Sverdrup, H.U., Boktr. John Griegds, The Norwegian North Polar Expedition with the 'Maud' 1918-1925, vol. 1a, no.5, Bergen, Norway, pp. 1-67.

Maykut, G.A., Untersteiner, N., 1971. Some results from a time-dependent thermodynamic model of sea ice. J. Geophys. Res. 76, 1550-1575.

McPhee, M.G., Kwok, R., Robins, R., Coon, M., 2005. Upwelling of Arctic pycnocline associated with shear motion of sea ice. Geophys. Res. Lett. 32, L10616.

Mellor, G.L., Blumberg, A.F., 1985. Modeling vertical and horizontal diffusivities with the sigma coordinate system. Month. Weather Rev. 113, 1380-1383.

Ono, N., 1967. Specific heat and heat of fusion of sea ice. In: Oura, H. (Ed.), Physics of Snow and Ice, vol. 1. Institute of Low Temperature Science, Hokkaido, Japan, pp. 599-610.

Perovich, D.K., Elder, B.C., 2001. Temporal evolution of Arctic sea-ice temperature. Ann. Glaciol. 33, 207-211.

Perovich, D.K., Elder, B.C., 2002. Estimates of the ocean heat flux at SHEBA. Geophys. Res. Lett. 29, 1344.

Perovich, D.K., Andreas, E.L., Curry, J.A., Eiken, H., Fairall, C.W., Grenfell, T.C., Guest, P.S., Intrieri, J., Kadko, D., Lindsay, R.W., McPhee, M.G., Morrison, J., Moritz, R.E., Paulson, C.A., Pegau, W.S., Persson, P.O.G., Pinkel, R., Richter-Menge, J.A., Stanton, T., Stern, H., Sturn, M., Tucker III, W.B., Uttal, T., 1999. Year on ice gives climate insights. Eos, Trans. Amer. Geophys. Union 481, 485-486.

Persson, P.O.G., Fairall, C.W., Andreas, E.L., Guest, P.S., Perovich, D.K., 2002. Measurements near the atmospheric surface flux group tower at SHEBA: near-surface conditions and surface energy budget. J. Geophys. Res. 107, 8045.

Pringle, D.J., Eicken, H., Trodahl, H.J., Backstrom, L.G.E., 2007. Thermal conductivity of landfast Antarctic and Arctic sea ice. J. Geophys. Res. 112, C04017.

Schmidt, G.A., Bitz, C.M., Mikolajewicz, U., Tremblay, L.-B., 2004. Ice-ocean boundary conditions for coupled models. Ocean Modell. 7, 59-74.

Semtner, A.J., 1984. On modelling the seasonal thermodynamic cycle of sea ice in studies of climatic change. Clim. Change 1, 27-37.

Turner, A.K., Hunke, E.C., Bitz, C.M., 2013. Two modes of sea-ice gravity drainage: a parameterization for large-scale modeling. J. Geophys. Res. 118, 2279-2294.

Untersteiner, N., 1961. on the mass and heat budget of Arctic sea-ice. Arch. Meteorol. Geophys. Bioklimatol. Ser. A 12, 151-182.

Vancoppenolle, M., Fichefet, T., Bitz, C., 2005. On the sensitivity of undeformed Arctic sea ice to its vertical salinity profile. Geophys. Res. Lett. 32, L16502.

Vancoppenolle, M., Fichefet, T., Goosse, H., Bouillon, S., Madec, G., Morales Maqueda, M., 2009. Simulating the mass balance and salinity of Arctic and Antarctic sea ice. 1. Model description and validation. Ocean Modell. 27, 33-53.

Vancoppenolle, M., Goosse, H., de Montety, A., Fichefet, T., Tremblay, B., Tison, J.-L., 2010. Modeling brine and nutrient dynamics in Antarctic sea ice: the case of dissolved silica. J. Geophys. Res. 115, C02005.

Vancoppenolle, M., Bouillon, S., Fichefet, T., Goosse, H., Lecomte, O., Morales Maqueda, M.A., Madec, G., 2012. LIM, The Louvain-la-Neuve Sea Ice Model. Note du Pole de modélisation, No 27, ISSN No 1288-1619, Institut Pierre-Simon Laplace (IPSL), France.

Washington, W., Parkinson, C.L., 2005. Introduction to three-dimensional climate modeling, second ed. University Science Books. 